\title{
Les adaptations à la volvation du squélette externe de la tête chez Caecosphaeroma burgundum Dollfus, Crustacé Isopode des eaux souterraines
}

\author{
par
}

C. MARVILLET*

\begin{abstract}
SUMMARY
The adaptations to volvation of the external cephalic skeleton of Caecosphaeroma burgundum Dollfus, a subterranean waters Isopod

The study of the cephalic capsule of Caecosphaeroma burgundum, a subterranean waters Isopod, demonstrates improved adaptations to volvation; these concern in a similar manner the other regions of the body, particularly the pleotelson.

From a primitive aquatic Isopod structure, the head of this blind Spheromid has been completely fashioned by many mechanical factors: posterior margin of pleotelson providing support on the head, relation of anterior angles of the second pereionit and, above all, the mandibuiar palps and antennae which retract into two deep grooves of the face.

The comparative study of the head of other volvational Isopods shows the importance of that "antennary factor", e.g. in Oniscoids, epigean Spheromids and some other subterranean waters Isopods (two Spheromids and one Cirolanid). This comparison shows that Caecosphaeroma burgundum is certainly the most specialized of all; it approaches perfection in volvation for it is the only one which rolls up into an hermetic sphere without outwards projections.

Volvation seems to play a two-fold role. It is a means of defense against predators used by single specimens and by copulating pairs, $\hat{\delta}$ and $q$ being then associated in two concentric spheres. Furthermore, it is a very important way for passive dissemination allowing settlement of these Crustacea in distant subterranean waters.
\end{abstract}

\section{MATERIEL ET TECHNIQUES}

Cette étude a pour objet principal Caecosphaeroma burgundum Dollfus, Isopode Sphéromien aveugle peuplant les eaux souterraines de Bourgogne et de Lorraine, pouvant se rouler en boule, mais d'autres Isopodes volvationnels y sont étudiés comparativement. Il s'agit de:

- Monolistra caeca Gerstaecker, autre Sphéromien aveugle des eaux sou-

\footnotetext{
* Laboratoire de Biologie Animale et Générale, Faculté des Sciences de la Vie et de l'Environnement, 6 boulevard Gabriel, 21000 Dijon, France.
} 
terraines (exemplaires provenant de la grotte de Podpeč en Yougoslavie)

- Faucheria faucheri Dollfus et Viré, Cirolanide hypogé et aveugle.

La répartition géographique de cet Isopode dans le Sud de la France est mainténant bien connue, grâce aux travaux de Bertrand (1974) et les individus étudiés ici ont été récoltés dans l'une des stations que signale cet auteur, la grotte de la Guiraudasso à Soulatgé, dans l'Aude.

- Deux espèces du Genre Sphaeroma Latreille, Sphéromiens épigés marins.

- Armadillo Latreille et Armadillidium vulgare Latreille, Oniscoïdes terrestres.

Les capsules céphaliques, décalcifiées et nettoyées à la potasse, sont colorées au noir de chlorazol. Quelques têtes de Caecosphaeroma hurgundum, ainsi préparées, sont incluses a la paraffine pour être sectionnées à la lame de rasoir suivant le plan sagittal et à plusieurs niveaux parasagittaux en tranches longitudinales verticales de quelques dixièmes de millimètre d'épaisseur (voir Figure 5 par exemple).

La compréhension des structures observées et l'élaboration de certaines figures ont été facilitécs par la réalisation d'une reconstitution spatiale de la tête, selon la méthode décrite par Chaudonneret (1967), à partir de coupes sériées colorées par un trichrome de type Prenant.

Les coupes sériées ont permis également de préciser la situation des insertions musculaires sur les parois squelettiques de la tête.

\section{A. Description de la tête de Caecosphaeroma burgundum}

\section{Les travaux antérieurs}

En ce qui concerne les Isopodes aquatiques en général et les Sphéromiens en particulier, peu d'auteurs se sont penchés sur l'anatomie détaillée de la tête, dont les caractéristiques liées à la volvation n'ont pratiquement pas été étudiées. Pour les espèces des eaux souterraines que nous avons examinées (Caecosphaeroma, Monolistra, Faucheria), seules les descriptions de Racovitza $(1910,1912)$ apportent quelques données, bien insuffisantes toutefois puisque cet auteur était plus orienté vers les caractères appliqués à la systématique que vers les détails du squelette céphalique. Gruner lui-même (1953) est resté très discret sur cet aspect descriptif de la tête dans son étude sur les mécanismes de la volvation.

Des travaux anatomiques plus précis sur la capsule céphalique concernent les Isopodes terrestres, volvationnels ou non (Jackson, 1926, 1928 - Vandel, 1943, 1960, 1962). Ils nous ont été précieux, malgré l'éloignement systématique de ces groupes. Nous y ferons souvent référence, ainsi qu'aux figures que Gruner $(1965,1966)$ donne de la tête des Isopodes aquatiques ou terrestres.

\section{Allure générale de la tête (Fig. 1 et 2)}

En grande partie dissimulée lorsque l'animal est enroulé (Fig. 4), la tête, to- 
talement aveugle, est large et globuleuse. En comparaison avec celle d'autres Isopodes, elle est profondément modifiée par la volvation, ainsi que nous le verrons, au niveau de la face et des régions latérales.

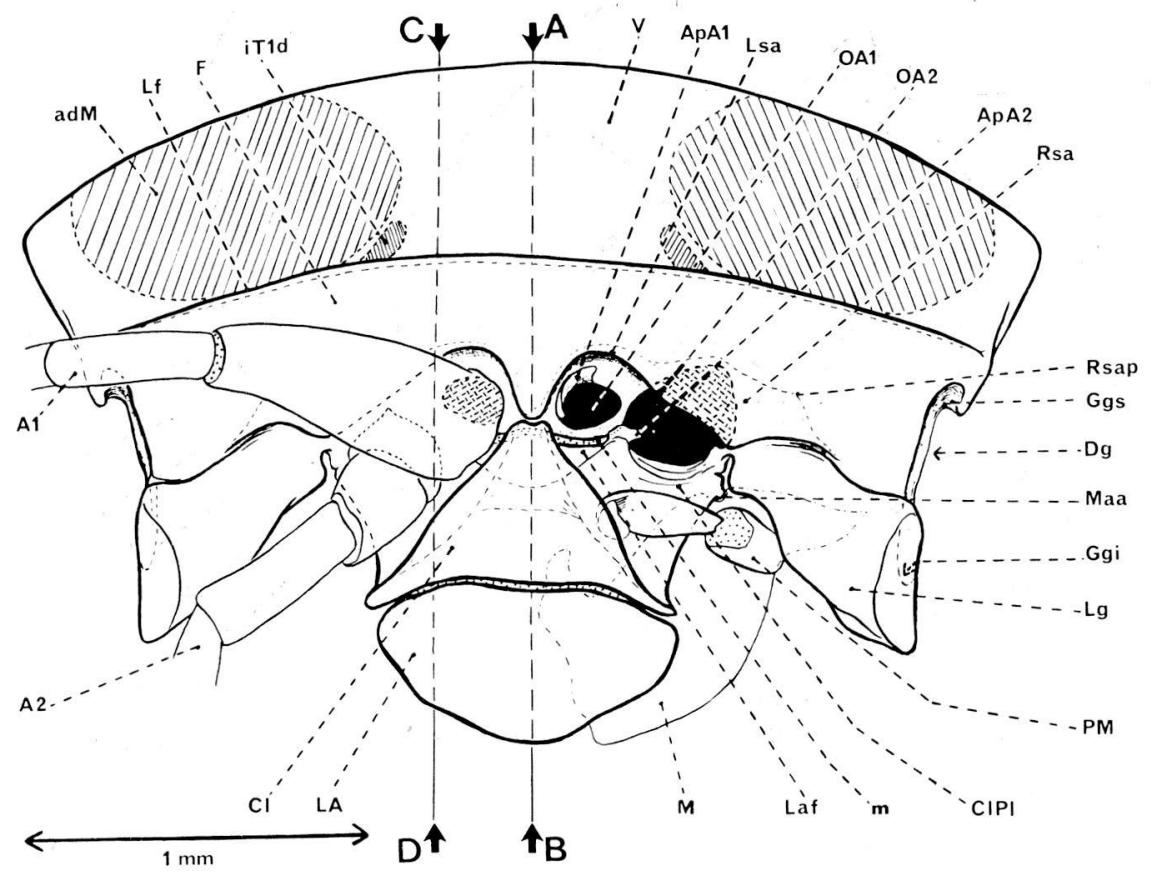

Fig. 1: Tête de Caecosphaeroma hurgunclum vue de face.

La mandibule gauche est en place et le palpe mandibulaire occupe sa position de repli au fond de la gouttière antennaire.

Le départ des deux antennes droites est figuré. Al a été dégagée de la gouttière antennaire.

La tranche verticale $\mathrm{AB}-\mathrm{CD}$ correspond a la figure 5.

\section{La face}

1. Les gouttières antennaires (Fig. I et 2)

L'allure originale de la face vient de ce que les orbites d'insertion des antennules et des antennes, ainsi que la région du front qui les porte, sont situées tout au fond de deux importantes gouttières obliques en direction dorso- 


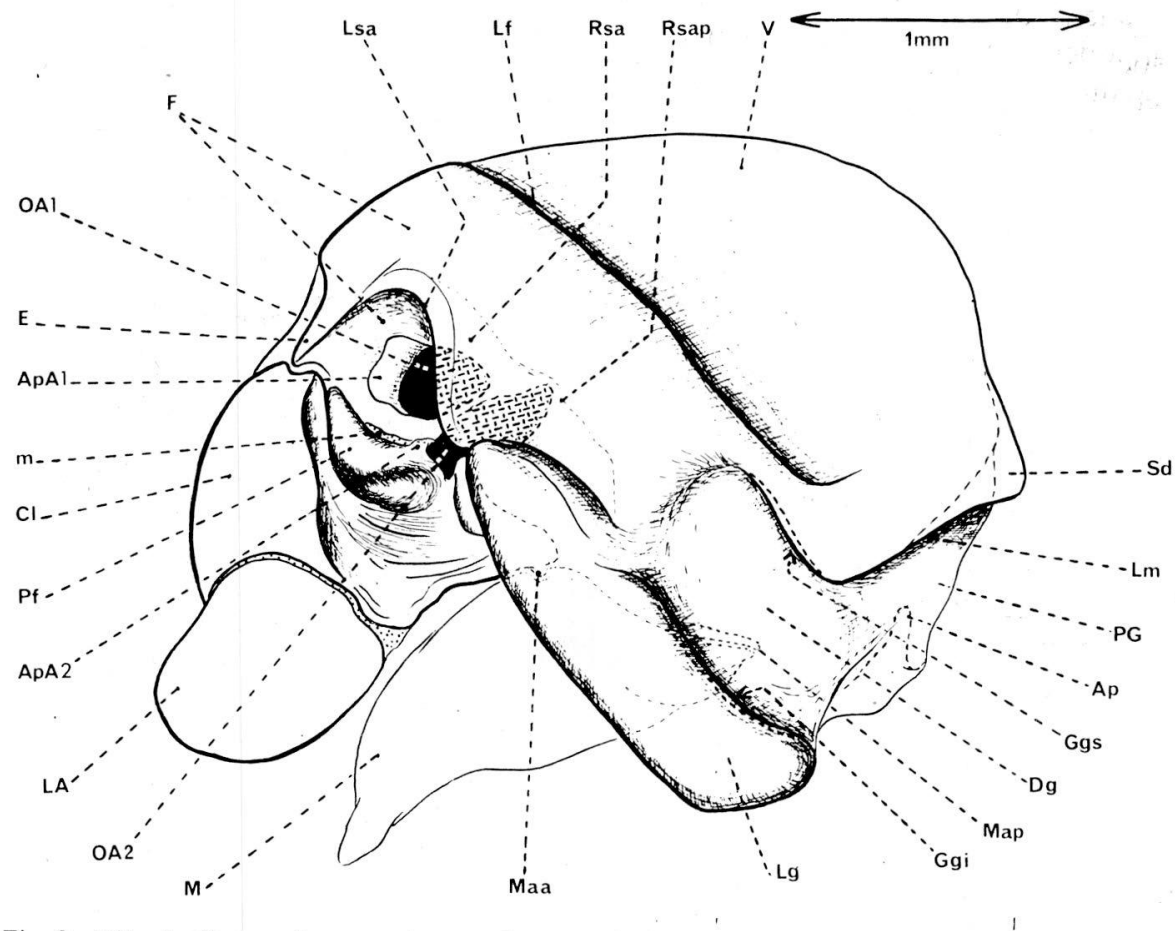

Fig. 2: Tête de Caecosphaeroma hurgundum vue de $3,4$.

L'articulation postérieure de la mandibule et l'apodème Ap sont visibles par transparence.

ventrale où peuvent se loger ces appendices et les palpes mandibulaires. Ces gouttières antennaires sont entourées d'un cadre très induré; leur creusement considérable et l'affrontement de l'écusson à la pointe dorsale du clypéus font que la face apparente est resserrée et réduite. Ce dispositif n'est pas sans évoquer celui que l'on observe chez les Trichoniscides (Vandel, 1943).

On y retrouve néanmoins, bien que très modifiés, les éléments traditionnels de la face des Isopodes, et plus particulièrement des Isopodes aquatiques (considérés comme plus primitifs): insertions des antennes proches de la ligne médiane, présence de deux fortes antennes de chaque côté, de lames génales bien développées, d'un processus frontal et d'une ligne supra-antennaire bien accusés. C'est, à peu de choses près, ce que l'on peut observer chez Sphaeromides ou Idotea par exemple.

2. Le clypéus et la lamina frontale (Fig. 1, 2 et 5)

Comme chez la plupart des Isopodes aquatiques (Jackson, 1926 - Vandel, 1943), une étroite bande membraneuse transversale (Fig. 1 et 2:m) sépare la 


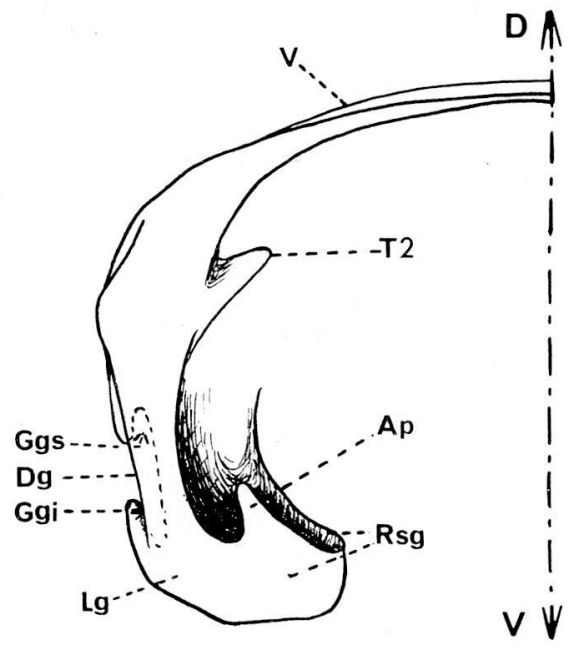

Fig. 3: Téte de Caecosphaeroma hurgundum. Foramen occipital (moitié gauche). L axe I) V correspond au plan sagittal.

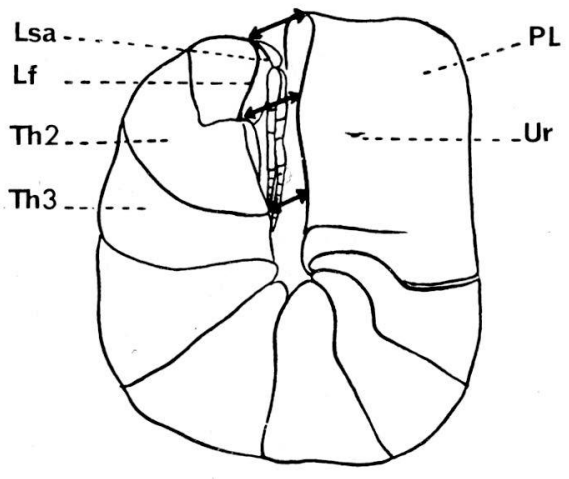

Fig. 4: Caecosphaeroma hurgundum enroulé. de profil. Le dessin est réalisé d'apris un animal mort légèrement entrebaillé. L'animal vivant enroulé est plus sphérique. Lès flèches indiquent la coaptation entre le bord du pléotelson d'une part et la ligne fiontale et les marges ventrales de $\mathrm{Th} 2$ et $\mathrm{Th} 3$ d'autre part. 


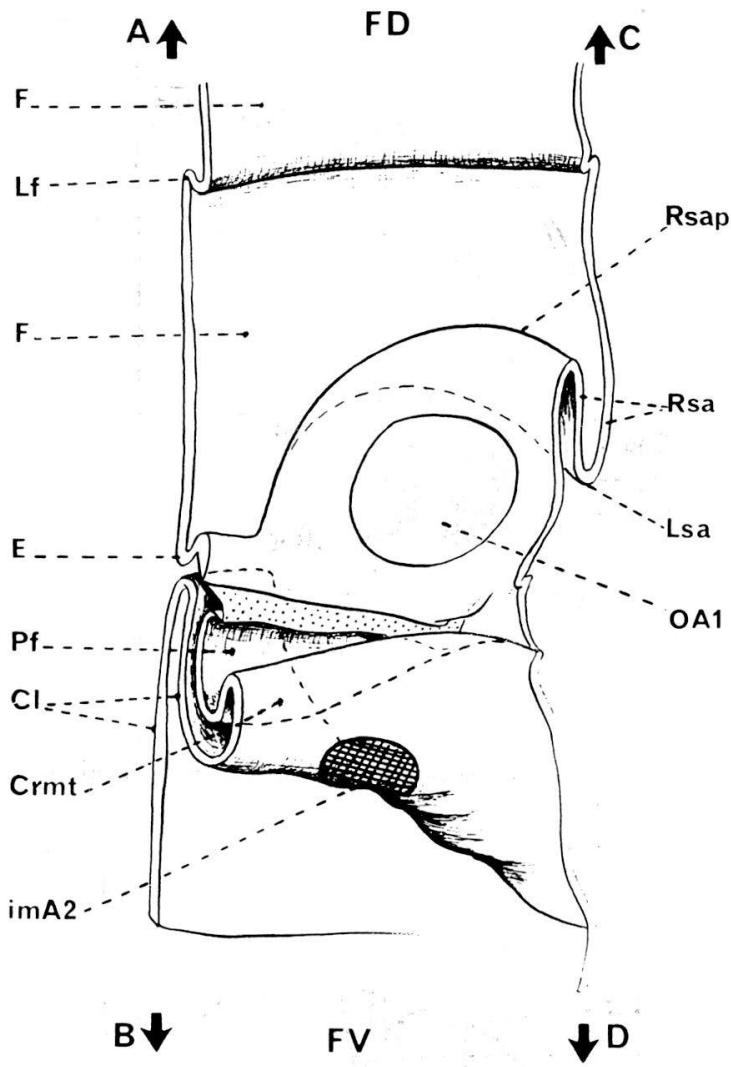

Fig. 5: Tête de Caecosphaeroma hurgundum. Coupe effectuée au niveau de ia face et correspondant à la tranche verticale $\mathrm{AB}-\mathrm{CD}$ portée sur la figure 1. Face interne.

$\mathrm{AB}$ passe légèrement à droite du plan sagittal.

Le labre n'est pas figuré. FD: face dorsale. FV: face ventrale.

partie du front qui porte les orbites d'insertion des antennes d'un ensemble plus ventral fait de deux éléments soudés, la lamina frontale et le clypéus.

$\mathrm{Vu}$ de face (Fig. 1), cet ensemble est étiré transversalement jusqu'aux processus latéraux du clypéus (ClPl), comparables aux lobes paraclypéaux des Insectes et qui portent les articulations antérieures des mandibules. de telle sorte qu'il constitue le plancher des gouttières antennaires. Le clypéus est très développé, élargi à sa base. Dorsalement, il atteint l'écusson de la ligne supraantennaire, à tel point que Racovitza (1912) éprouvait le besoin de préciser: "clypéus non soudé à l'écusson". 
Vu de profil (Fig. 2), cet ensemble révèle son épaisseur considérable et laisse apercevoir le creusement du clypéus ainsi que le fort processus médian (Pf) de la lamina frontale. C'est grâce à une coupe à peu près sagittale (Fig. 5), ou sur un modèle en relief, que l'on comprend réellement l'enroulement de cette région de la face qui forme l'armature médiane entre les deux gouttières antennaires. Ainsi que le note Snodgrass (1952) chez Ligyda, le clypéus de Caecosphaeroma burgundum apparaît comme une émergence antérieure de la lamina frontale dont l'extension est indiquée sur la figure 5 par la situation de l'insertion des muscles de l'antenne (imA2). La lamina frontale est en effet considérée habituellement comme le sternite du segment antennaire chez les Isopodes, ce qui, de toute évidence, est en partie exact. Par contre, interpréter le clypéus, suivant l'opinion couramment admise, comme le sternite du segment mandibulaire, nous paraît impensable puisqu'aucun muscle appendiculaire des mandibules ne s'y insère.

Cette même figure 5 permet également d'apprécier à quel point la soudure lamina frontale - clypéus développe un fort repli chitineux interne, véritable tablette transversale de renforcement de la face située juste en dessous de la région de moindre résistance des orbites d'insertion des antennes. Cette crête s'étend ainsi entre les articulations antérieures des mandibules et joue exactement le même rôle que la suture épistomienne, ou clypéo-frontale, des Insectes. Snodgrass (1952) la décrit sans lui donner de nom; nous l'appellerons "crête intermandibulaire transverse" (Crmt). Elle correspond à la structure que Jackson (1926) décrit chez Ligia par le terme impropre de "barre maxillaire".

Ainsi le clypéus, profondément transformé, replié en profondeur, étiré transversalement, conserve ses deux caractéristiques fonctionnelles fondamentales: il supporte les articulations antérieures des mandibules et, corrélativement, présente une crête transversale interne. Cette crête de renforcement de la face se poursuit de chaque côté par le rebord interne du repli du lobe génal (Rsg, Fig. 3 et 9) tout comme, chez les Insectes typiques, la suture épistomienne se prolonge latéro-postérieurement par la suture sub-génale.

\section{Front, ligne supra-antennaire, ligne frontale et toit de la capsule céphalique (Fig. I et 2)}

Limité en avant par la ligne supra-antennaire, le toit crânien est divisé par la ligne frontale ( $\mathrm{Lf}$ ) en un front antérieur et un vaste vertex postérieur, occupé en majeure partie par l'insertion des muscles mandibulaires $(\operatorname{ad} M)$ et dépourvu de toute trace dorsale du segment des maxillipèdes.

La ligne supra-antennaire est d'autant plus accusée qu'elle surplombe les gouttières antennaires (Fig. 1, 2 et 5: Rsa) et qu'elle développe un fort écusson médian. Morphologiquement très importante selon les auteurs, car elle marquerait la limite entre les tergites et les sternites des segments céphaliques, elle divise le front en deux parties: l'une postérieure, plus dorsale et vraisemblablement tergale, limitée en arrière par la ligne frontale, l'autre antérieure 
et pleuro-sternale, plus ventrale, qui porte les orbites d'insertion des antennes. Ces deux régions du front sont appelées respectivement profrons et postfrons par certains (Jackson, 1926, chez Ligia - Vandel, 1943, chez Ligidium).

Le terme "épistome", fréquemment utilisé dans les descriptions de la face, est ambigü car il désigne des structures très différentes selon les auteurs, mëme récents. C'est ainsi que, à la suite de Racovitza (1912), certains (Hennequin, 1934 - Holthuis, 1956) désignent par épistome la partie de la face située sous la ligne supra-antennaire et portant les orbites d'insertion des antennes alors que, dès 1926, Jackson proposait de rejeter ce terme en insistant sur la confusion due à son emploi différent selon que l'on s'adresse aux Isopodes ou aux Décapodes. Vandel (1943) le rejette à son tour.

D’autres réservent le nom d’épistome au clypéus: Snodgrass (1952) par exemple. chę Ligia, tout en reconnaissant que "l'épistome des Isopodes est la réplique exacte du clypéus des Insectes", ce qui aurait dú le conduire à adopter le mème terme dans ces deux groupes, ou Lomoardo (1975) chez Anilocra.

Il nous paraît donc opportun d'abandonner toute utilisation du mot épistome. non seulement pour désigner le clypéus, mais également toute autre région de la face des Isopodes.

De profil, la ligne supra-antennaire (Fig. 2, Lsa) apparaît comme une importante limite anatomique qui oppose franchement la face au toit de la capsule céphalique, à tel point que Racovitza (1910) l'appelle "rebord frontal". Cet auteur donne à l'écusson le nom de "processus frontal" (chez Faucheria), mais il faut le rejeter car il prête confusion avec le processus médian de la lamina frontale.

La ligne frontale qui, de par sa position, mériterait plutôt le nonı de "postfrontale", réunit typiquement le bord antérieur des yeux de nombreuses espèces oculées. Elle est souvent figurée sans que l'on puisse juger de son développement en profondeur (Racovitza, 1910 - Hennequin, 1934 - Gruner, 1953) ou même comme une simple ligne peu accusée (Jackson, 1926 - Manton, 1964). Chez Caecosphaeroma burgundum et les autres Sphéromiens examinés, elle prend l'allure d'un repli bien net (Fig. 5 et 9). Appelée quelquefois "crête occipito-frontale" (Racovitza, 1910 - Hennequin, 1934), elle est caractérisée par le fait que, juste à son niveau et un peu en arrière d'elle, s'insèrent les deux prolongements dorsaux du tentorium antérieur (les "processus pharyngiens" de Jackson), comme le montre la figure l (iTld). Dans le cas de Faucheria (Fig. 6) et de tous les Isopodes où la ligne frontale est absente, ces insertions marquent son emplacement.

Si l'on se fie uniquement à la position des insertions dorsales des muscles mandibulaires sur le toit céphalique (Fig. 1, adM et Manton, 1964), la ligne frontale semble représenter la limite antérieure du segment mandibulaire, mais ce n'est pas là une preuve suffisante. Jackson (1928) signale que, chez certains Isopodes terrestres, une partie de ces insertions peut être située en avant d'elle. Dans ce cas, la ligne frontale n'est pas une limite morphologique, mais plutôt une ligne de renforcement à signification purement mécanique, ce qui est particulièrement évident, comme nous le verrons, chez Caecosphaeroma burgundum et les Sphéromiens. 


\section{Les flancs de la capsule céphalique (Fig. 2)}

Ils ont également subi des modifications considérables dont nous envisagerons plus loin les rapports évidents avec la volvation.

On y voit une vaste dépression génale à fond plat (Fig. 1, 2, 3 et 9: Dg), entre un lobe génal antéro-ventral très développé $(\mathrm{Lg})$ et une région postgénale surmontée d'une ligne en surplomb $(\mathrm{Lm})$ qui délimite la postgéna et le vertex. Par transparence, on peut repérer la position particulière de l'articulation postérieure de la mandibule (Marvillet et Chaudonneret, 1975) et celle de l'apodème latéro-ventral Ap sur lequel s'attachent les bras sclérifiés du squelette maxillo-sternal (Marvillet, 1972).

Le lobe génal correspond en gros à la lame génale des Isopodes aquatiques telle que Gruner (1965) la figure chez Idotea et dont la présence est considérée par Vandel (1943) comme un caractère primitif. Dans sa partie la plus dorsale, il s'insinue sous le repli supra-antennaire et se continue par la partie du plancher de la gouttière antennaire qui porte les orbites d'insertion des antennes. Son extension vers l'avant forme le bord latéro-ventral de la gouttière antennaire (Fig. 1) et, en arrière, son reploiement vers l'intérieur donne naissance au repli subgénal (Fig. 3 et 9: Rsg). Son bord postéro-dorsal est entaillé en une gouttière (Ggi) où peut s'insinuer une crête du péréionite 2.

La ligne en surplomb $(\mathrm{Lm})$ correspond à la ligne marginale des autres Isopodes, comme en témoigne la comparaison avec des Sphéromiens oculés (Fig. 8) où cette ligne coïncide avec les bords postérieur et latéral de l'oeil. Chez de nombreux Isopodes, où la ligne supra-antennaire est bien nette, la ligne marginale la rejoint en avant de la tête, à l'endroit où aboutit également la ligne frontale quand elle existe. L'extension de la dépression génale (Dg) de Caecosphaeroma burgundum et l'intervalle apparu entre la ligne supraantennaire et la ligne frontale font que ligne marginale et ligne supra-antennaire ne se rejoignent pas.

Dans sa partie la plus antérieure, la ligne marginale remonte légèrement, à angle droit, en direction dorsale. Elle court alors parallèlement à l'extrémité de la ligne frontale. Le bourrelet saillant compris entre les deux voit son relief accentué à la fois par la goùttière supérieure de la dépression génale (Ggs) et par le repli de la ligne frontale ( $\mathrm{Lf})$ comme l'explique la figure 9.

\section{Le segment des maxillipedes}

On sait que, chez les Isopodes, le segment des maxillipèdes est annexé par la capsule céphalique. On admet, avec beaucoup de vraisemblance, que la limite postérieure de la tête proprement dite est matérialisée, chez la plupart d'entre eux, par une suture occipitale dorsale qui s'étend dans la région postérieure du vertex et se poursuit latéralement sur les aires postgénales par deux sillons verticaux.

Si l'on ajoute à cela le découpage du squelette ventral maxillo-sternal par des bras intersegmentaires sclérifiés, l'ensemble du segment des maxillipèdes 
apparaît, ainsi que le souligne Jackson (1926) comme un anneau continu situé à l'arrière de la capsule céphalique et faisant suite à la tête sensu stricto.

La persistance de ces sillons dorsal et latéraux serait un caractère primitif propre aux Isopodes aquatiques (Racovitza, 1912 - Jackson, 1928). Certains Cirolanides, Sphaeromides par exemple, tendent à le prouver. A partir de ce dispositif, une évolution au sein des Isopodes fait que:

- Le segment des maxillipèdes va se réduire.

- Sa limite antérieure visible extérieurement tend à disparaître, la partie dorsale de la suture occipitale étant la première affectée par cette régression. Elle est encore visible chez les Oniscoïdes primitifs (Ligia, Ligidium) mais pas chez les Oniscoïdes plus évolués (Jackson, 1928 - Vandel, 1960) qui conservent par contre ses parties latérales, bien apparentes, comme nous l'avons vérifié sur de nombreuses espèces.

Caecosphaeroma burgundum, les autres Sphéromiens observés (Caecosphaeroma virei, Monolistra caeca, Sphaeroma hookeri, Sphaeroma serratum) et certains Cirolanides (Faucheria faucheri), tous aquatiques et volvationnels, ont perdu toute trace de limite du segment des maxillipèdes extérieurement visible sur la capsule céphalique. Seule leur armature céphalique ventrale révèle encore les indices de la segmentation (Marvillet, 1972).

Des expansions chitineuses creuses s'invaginent à partir des portions latérales de la suture occipitale. Ces formations, décrites par Jackson (1926) sous le nom de "tergal alae", ont conservé ce nom, même dans la littérature récente, avec un certain nombre de variantes: "ailes des apodèmes tergaux" par exemple (Lombardo, 1975).

Même dans les cas où la suture occipitale régresse, ces ailes tergales sont toujours présentes. C'est ainsi que Tait (1917) les décrit chez Glyptonotus où, bien sûr, le dispositif est différent, puisque les deux premiers segments thoraciques sont incorporés à la capsule céphalique, mais où le péréionite 1 est réduit à une mince bandelette en croissant coincée entre la tête et le péréionite 2 .

Dans le cas d'Armadillo, où le segment des maxillipèdes n'est plus représenté que par une marge postérieure étroite des postgénas, ces expansions internes sont également présentes et l'on peut y insinuer une pointe mousse à partir du sillon externe d'invagination.

Certains Sphéromiens (Sph. hookeri et Sph. serratum) et Faucheria faucheri ont des ailes tergales bien développées, bien qu'extérieurement rien ne signale la présence d'une segmentation. Par contre, chez Caecosphaeroma et Monolistra, ces formations sont elles-mêmes tellement réduites et refoulées sur la marge postérieure de la tête, que leur assimilation aux ailes tergales des auteurs serait hasardeuse s'il n'y avait les insertions musculaires pour trancher la question. Holmgren (1920) chez Asellus, Jackson (1926) et Manton (1964) chez Ligia, Schmalfuss (1974) chez Mesidotea, Ligia et Tylos, décrivent sur les ailes tergales les insertions de muscles provenant des antennes et des pièces buccales. Les expansions de Caecosphaeroma burgundum, malgré leurs dimensions réduites, donnent insertion à certains muscles des antennes, des man- 
dibules, des maxilles et des paragnathes. Elles correspondent donc aux ailes tergales des auteurs.

Le fait que ces ailes tergales reçoivent des muscles antennaires et des muscles des diverses pièces buccales permet de penser qu'il s'agit en réalité de formations de signification complexe, dues probablement à la coalescence de plusieurs limites intersegmentaires (comme, par exemple, le bras antérieur du tentorium d'Insecte). Nous leur donnerons le nom de tentorium postérieur (Fig. 3, T2), par opposition au vaste tentorium antérieur dont nous avons décrit les orifices d'invagination dans un travail précédent (Marvillet, 1972). La suture occipitale serait alors quelque chose de beaucoup plus complexe qu'une simple limite intersegmentaire.

\section{B. Les structures liées à la volvation}

La faculté de se rouler en boule est un phénomène assez répandu dans le monde animal et tout particulièrement chez les Isopodes, à tel point que Vandel (1959) voit dans cette aptitude une "tendance de groupe" de ces Crustacés. Chez les Oniscoïdes, plusieurs lignées aboutissent à des formes volvationnelles. Les Cirolanides connaissent la même évolution et la volvation devient la règle générale chez les Sphéromiens, ce qui fait des eaux souterraines un milieu riche en espèces capables de se conformer en une sphère à peu près parfaite.

A la volvation correspondent un certain nombre d'adaptations anatomiques: forme générale du corps, bombement des tergites, coaptation des segments thoraciques entre eux, escamotage des antennes, application du bord postérieur du pléotelson sur la tête, élimination des saillies extérieures à la boule. Nous ne reviendrons pas sur ces exigences mécaniques générales, étudiées par de nombreux auteurs, dont Racovitza (1910, 1912), Vandel (1943, 1960, 1962), Gruner (1953), Hennequin (1934).

Au niveau de la capsule céphalique par contre, il est intéressant de préciser à quel point la volvation peut bouleverser les éléments anatomiques préexistants et de comparer les structures rencontrées chez Caecosphaeroma burgundum et les Sphéromiens avec les dispositifs tout aussi efficaces mais radicalement différents adoptés par les Cirolanides et les Oniscoïdes supérieurs.

\section{La forme de la tête}

L'aspect globuleux de la tête est souvent considéré comme lié à la volvation. Nous n'y voyons pour notre part ni relation obligatoire, ni même règle générale. Certes les Sphéromiens, malgré une ligne marginale très saillante, ont une tête globuleuse, mais ce n'est pas du tout le cas, par exemple, des Oniscoïdes volvationnels Armadillidium ou Armadillo. Comme le souligne Jackson (1928), la tête de ces derniers est au contraire tassée dans le sens antéro-posté- 
rieur et l'occiput comme les génas ont la forme d'une bande étroite. Tout au plus la capsule céphalique est-elle bombée dorsalement, mais la face est large, aplatie et brutalement différenciée des régions dorsales par un angle bien marqué. La ligne marginale, qui avait disparu, est remplacée par une ligne marginale secondaire saillante. En réalité, chez ces Oniscoïdes, la tête n'est pas tant adaptée à la volvation qu'à la protection des antennes qui s'appliquent contre la face (Gruner, 1953). La capsule céphalique du Cirolanide Faucheria faucheri correspond assez à cette description.

Il en est tout autrement de Caecosphaeroma et des Sphéromiens: l'opposition vertex-face est estompée, malgré le relief de la ligne supra-antennaire qui sépare radicalement les deux. C'est que l'ensemble clypéus-labre, saillant en avant, est bombé suivant une courbe qui prolonge celle des régions dorsales de la tête (Fig. 2). Il faut en chercher l'explication dans le creusement des deux gouttières antennaires en direction ventrale et dans le développement corrélatif de leur armature médiane commune, le clypéus.

Lorsque l'animal est enroulé, cette face bombée vient se loger entièrement dans l'évidement correspondant du pléotelson, fortement voussuré (Fig. 4). On peut considérer d'ailleurs que les antennes des Oniscoïdes volvationnels ou de Faucheria occupent un volume équivalent dans la boule, malgré la petitesse du clypéus.

Faut-il enfin attribuer la régression du segment des maxillipèdes à la volvation? Rien ne le prouve, puisqu'il est présent chez les Oniscoïdes capables de s'enrouler, malgré le tassement antéro-postérieur de la tête. Peut-être cette régression ne se produit-elle qu'au stade ultime de l'évolution "volvationnelle", les Sphéromiens et les Cirolanides atteignant la quasi-perfection à ce point de vue. On peut tout aussi bien n'y voir qu'une tendance évolutive indépendante de la volvation dans certains groupes d'Isopodes aquatiques.

\section{La coaptation du pléotelson et de la tête}

Lorsque Caecosphaeroma burgundum s'enroule, le bord postérieur arrondi et uni du pléotelson vient s'appliquer étroitement sur la ligne frontale qui joue le rôle d'un bourrelet de renforcement transversal dans le vaste vertex soumis de surcroît aux tractions des puissants muscles adducteurs des mandibules (Fig. 1 et 4).

La coaptation est d'autant plus parfaite que la ligne frontale est homogène, sans rupture et qu'elle est naturellement prolongée de chaque côté par les bords antérieurs des péréionites 2 et 3 sur lesquels s'appuient les marges latérales du pléotelson (Fig. 4, 7 et 8). Chez d'autres Isopodes volvationnels l'appui du pléotelson se fait sur la ligne supra-antennaire (Faucheria, Tylos, Helleria,...) et la ligne frontale est alors absente, comme c'est le cas général des Isopodes aquatiques non susceptibles de se rouler en boule.

La présence de la ligne frontale chez Caecosphaeroma burgundum et les autres Sphéromiens semble donc directement liée aux impératifs de la volvation. Elle assure une fermeture plus hermétique de la boule que la ligne supra- 
antennaire interrompue par l'écusson et sa position, postérieure à cette dernière, fait que la tête est dissimulée plus profondément sous le pléotelson.

\section{L'écusson}

C'est une formation triangulaire à pointe antérieure médiane qui peut quelquefois se développer en un vaste bouclier transversal tel qu'on l'observe chez les Eubelidae. Il vient immédiatement à l'esprit que cet écusson est un renforcement de la ligne d'appui du pléotelson chez les formes volvationnelles puisqu'il est généralement présent, soit sur la ligne supra-antennaire lorsqu'elle joue ce rôle (Faucheria par exemple), soit sur la ligne frontale ou sur une ligne postérieure néoformée, la ligne post-scutellaire, lorsque la ligne supra-antennaire a été incorporée à la face (Oniscoïdes volvationnels évolués).

Cette opinion généralement admise ne tient pas compte de plusieurs faits qui la contredisent:

La formation d'un écusson frontal médian est une tendance fréquente de nombreux Isopodes ne se roulant pas en boule (Ligia, Ligidium, Oniscus, Trichoniscus...).

- Cet écusson peut être au contraire discret (Tylos) ou même absent (Armadillo) chez des formes volvationnelles.

- Dans le cas de Caecosphaeroma burgundum, il est au contraire bien développé, mais ce n'est pas la ligne d'appui du pléotelson, la ligne frontale, qui le porte, mais la ligne supra-antennaire plus antérieure, largement dissimulée sous le bord postérieur du corps une fois l'animal enroulé!

Chez les Sphéromiens, l'écusson est donc avant tout une structure de renforcement de la face puisqu'il ne représente que l'épanouissement dorsal d'une sorte de cloison verticale médiane dressée entre les gouttières antennaires (Fig. 2 et 7). Cette interprétation vaut également pour Faucheria (Fig. 6) où pourtant l'écusson est situé juste sur la ligne d'appui du pléotelson.

\section{Les adaptations corrélatives du pléotelson}

Les Isopodes et les Diplopodes volvationnels améliorent la coaptation de l'extrémité du corps sur la tête grâce à deux adaptations de l'abdomen ou du pléotelson:

- Un bord postérieur continu permettant une fermeture aussi hermétique que possible.

- Un évidement interne où se loge la tête et dont l'efficacité est accrue chez les Sphéromiens par la réduction du volume des pléopodes (Racovitza, 1910).

La disparition des vides entre les pièces qui participent à la constitution du bord postérieur du corps et à la formation d'une ligne sans faille est un point d'évolution important conduisant à une volvation parfaite. Le Myriapode Glomeris y parvient par son bouclier préanal, les Oniscoïdes volvationnels 
par des modifications variées, soit des uropodes, soit du telson. Helleria brevicornis (Gruner, 1953 - Mead, 1963) va plus loin dans la réduction du nombre de pièces visibles de l'extérieur, puisque les uropodes ne sont plus apparents et que le telson trapézoidal subsiste seul.

Parmi les Isopodes aquatiques volvationnels, le genre Caecosphaeroma pousse la simplification à l'extrême: alors que les uropodes en forme de sabre de Monolistra, ou en palettes de Faucheria et des Sphéromiens, font encore saillie à l'extérieur de l'animal enroulé, ils sont vestigiaux chez Caecosphaeroma virei et ont à peu près disparu chez $C$. burgundum (Fig. 4) où le pléotelson n'est plus qu'une pièce monolithique bombée au bord postérieur arrondi et sans faille.

Cette simplification anatomique favorise une coaptation parfaite, ce qui n'est pas le cas d'espèces volvationnelles pourtant très évoluées, comme $A r$ madillidium ou Armadillo, chez lesquelles le bord postérieur du pléotelson, bien que très modifié, est fait de nombreux éléments. Entre lui et la tête persiste une fente par laquelle l'écusson reste visible quand l'animal est enroulé.

Hennequin (1934) considérait Sphaeroma serratum comme un type volvationnel presque parfait. Caecosphaeroma burgundum est beaucoup plus evolué dans ce sens et, comme Racovitza (1910) le disait d'après l'allure générale du corps: "Les Monolistrini arrivent certainement bien près de la perfection dans leur enroulement. Toute leur morphologie externe est influencée par cette tendance à devenir à volonté une boule parfaite."

\section{Le reploiement des antennes}

Les Isopodes volvationnels ont deux façons d'escamoter leurs antennes:

- Les exoantennés développent un système de gouttières en direction postérieure sur la surface dorsale de la tête et du premier péréionite libre. L'animal une fois enroulé, les antennes sont encastrées dans ces gouttières mais restent entièrement visibles de l'extérieur.

- Les endoantennés les logent à l'intérieur de la boule dans des gouttières développées soit en direction latérale sur la face puis sur une partie des génas (Faucheria pour les Cirolanides, Fig. 6 - Armadillo par exemple, pour les Oniscoïdes), soit en direction ventrale sur la face seule (Sphéromiens).

Les gouttières sont accompagnées dans tous les cas de butées et de saillies qui maintiennent les antennes en place et les protègent.

Les gouttières antennaires ont puissamment modelé la face de Cuecosphaeroma burgundum. Chacune d'elles accueille, lors de la volvation, du fond vers la surface, le palpe mandibulaire, l'antenne puis l'antennule. Les trois sont alors solidement encadrés sur les côtés et dorsalement (Fig. 1) par un ensemble fait d'une armature médiane, d'un surplomb dorsal et d'un lobe génal latéral (le pléotelson venant clore la gouttière à l'extérieur).

\section{a. L'armature centrale entre les gouttières antennaires}

Nous avons décrit les trois éléments qui la constituent de haut en bas: l'écus- 


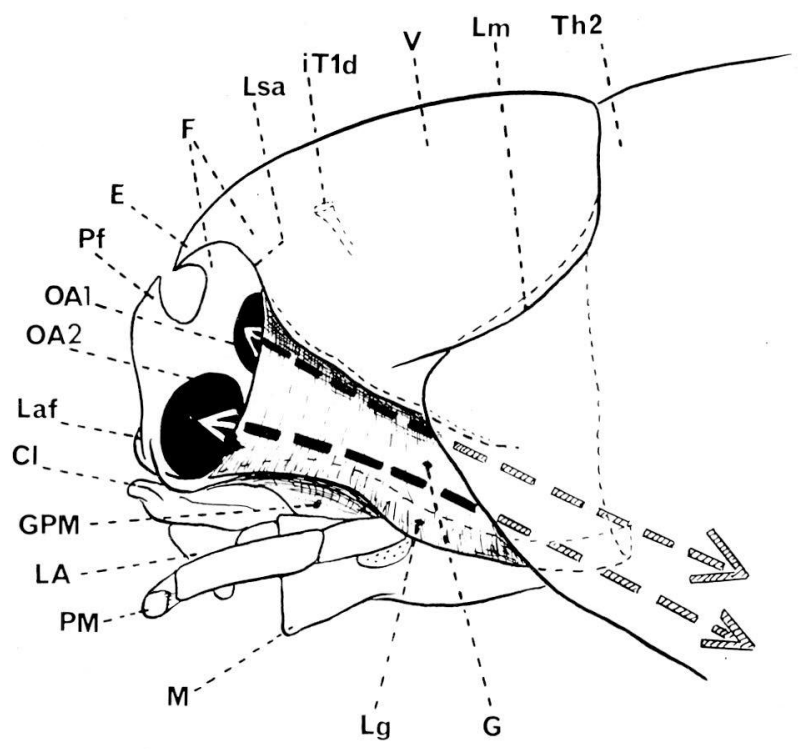

Fig. 6: Tẻte de Faucheria faucheri. Schéma, de profil.

Les deux flèches en tirets indiquent la position des antennes lorsque l'animal est enroulé.

son, dont nous avons évoqué la signification, le processus frontal et le clypéus (Fig. 2).

+ Le processus frontal des Isopodes est typiquement une émergence de la lamina frontale en direction médio-dorsale, juste entre les orbites antennaires. A quelques exceptions près (Tylos, Helleria), il est peu développé là où ces orbites sont écartées (Isopodes terrestres), mais il l'est beaucoup plus lorsqu'elles sont rapprochées de la ligne médiane (Isopodes aquatiques, Idotea par exemple, Fig. de Gruner, 1965). Il joue alors le rôle d'une structure de renforcement.

Dans la lignée des Cirolanides, le processus frontal prend une grande extension (Typhlocirolana, Sphaeromides). Il atteint son apogée chez l'espèce volvationnelle des eaux souterraines Faucheria faucheri, sous la forme d'une cloison verticale médiane qui s'unit largement à la formation analogue dressée sous l'écusson et qui développe un bec dorsal en direction de la pointe de celui-ci (Fig. 6).

On ne peut que rapprocher l'allure de ce dispositif de celui que réalise Caecosphaeroma burgundum, mais où le processus frontal, malgré son importance, est relégué au second plan par un clypéus extraordinairement puissant. 
+ Le clypéus est normalement plat et peu saillant chez les Isopodes aquatiques et les Oniscoïdes inférieurs. Dans quelques cas (Idotea par exemple) il annonce par son importance celui des Sphéromiens. Dans ce groupe, le grand développement de sa région centrale qui s'étale et recouvre ses deux flancs évidés pour le logement des antennes en fait un cas unique parmi les Isopodes. Son rôle de renforcement mécanique entre les deux gouttières antennaires peut seul expliquer sa taille considérable.

L'ensemble de cette armature des Sphéromiens rappelle l'excroissance médiane des exoantennés, allongée entre les deux gouttières antennaires sur le front et le vertex et qui joue le même rôle pour le logement des antennes.

\section{b. Le surplomb dorsal}

L'antennule est insérée au fond de la gouttière antennaire et la forme coudée de son premier article lui permet de se déployer facilement par un simple mouvement de pivot. L'antenne est insérée plus latéralement et sa base est en partie cachée par le repli supra-antennaire qui recouvre aussi la partie tout à fait antérieure du lobe génal (Fig. I et 2). Un surplomb aussi accusé est exceptionnel ches les Isopodes et doit être attribué à l'importance de la gouttière antennaire, à l'enfoncement corrélatif de la face sous la ligne supraantennaire (Fig. 5) ainsi qu'à la taille considérable des deux antennes.

\section{c. Le lobe génal}

C'est également par le creusement de la gouttière antennaire qu'il faut expliquer son ampleur ainsi que, par voie de conséquence, la migration vers l'intérieur de la marge ventrale primitive de la géna qui forme le repli subgénal. L'articulation postérieure de la mandibule suit ce mouvement et le dispositif fait alors penser à une esquisse d'entotrophie (Marvillet et Chaudonneret, 1975). On le retrouve à peu près tel quel chez Monolistra. Sphaeroma et Faucheria. Cette dernière espèce toutefois n'a pas un lobe génal aussi développé que celui de Caecosphaeroma, puisque la gouttière dorso-ventrale n'est pas creusée en profondeur pour abriter les antennes qui se replient dans une autre direction et qu'elle ne sert qu'au logement du palpe mandibulaire (Fig. 6, GPM).

\section{La coaptation de la tête et du deuxième segment thoracique}

La capsule céphalique de Caecosphaeroma burgundum est profondément encastrée dans le deuxième segment thoracique (Fig. 4, Th2). La mobilité de l'une par rapport à l'autre qui, de ce fait, est déjà limitée, est encore considérablement réduite au cours de la volvation par des dispositifs d'engrenage et de verrouillage (Fig. 7 et 9). Il en est de même chez les Sphéromiens marins oculés (Sphaeroma, Fig. 8). 


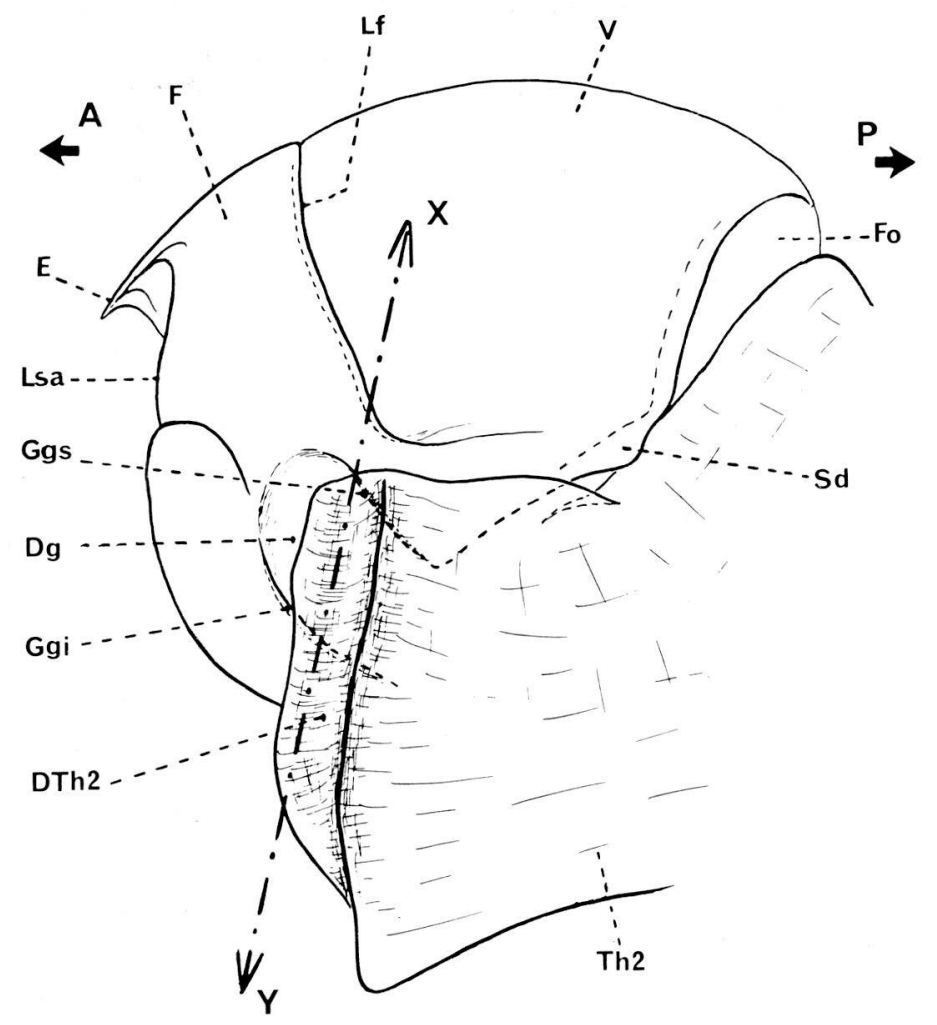

Fig. 7: Tête de Caecosphaeroma hurgundum, de profii. Schéma expliquant la coaptation de la capsule céphalique et du péréionite 2 .

La coupe XY correspond à la figure 9 .

On voit que la ligne frontale est prolongée vers le bas par une crète du bord antérieur de Th2.

La dépression DTh2 est peu accusée chez les Sphéromiens. A: région antérieure. B: région postérieure.

Deux saillies tout à fait antérieures du péréionite 2 encadrent la tête (Fig. 4,7 et 8 ). Chacune d'elles présente à sa base une semelle qui glisse sur la dépression génale (Fig. 9, Dg) et dont les crêtes latérales (CrTh2) s'insinuent dans les gouttières génales supérieure et inférieure (Ggs et Ggi). En même temps, comme le montrent les figures 7 et 8 , les marges latéro-postérieures de la capsule céphalique s'encastrent dans le bord antérieur du premier segment thoracique libre: la partie tout à fait latérale du vertex, limitée par la ligne marginale $(\mathrm{Lm})$, est recouverte par le rebord dorsal de la saillie antérieure du péréionite 2 alors que, juste en arrière, c'est l'angle occipital postérieur du vertex $(\mathrm{Sd})$ qui chevauche le péréionite. 
Malgré quelques modifications, les aires génales des Isopodes terrestres volvationnels évolués (Armadillidium, Armadillo) n’ont pas subi un tel bouleversement et sont assez peu modifiées en comparaison avec celles d'Oniscoïdes ne se roulant pas en boule (Porcellio par exemple). Leur géna n'est en effet que partiellement consacrée au glissement de la saillie antérieure du péréionite

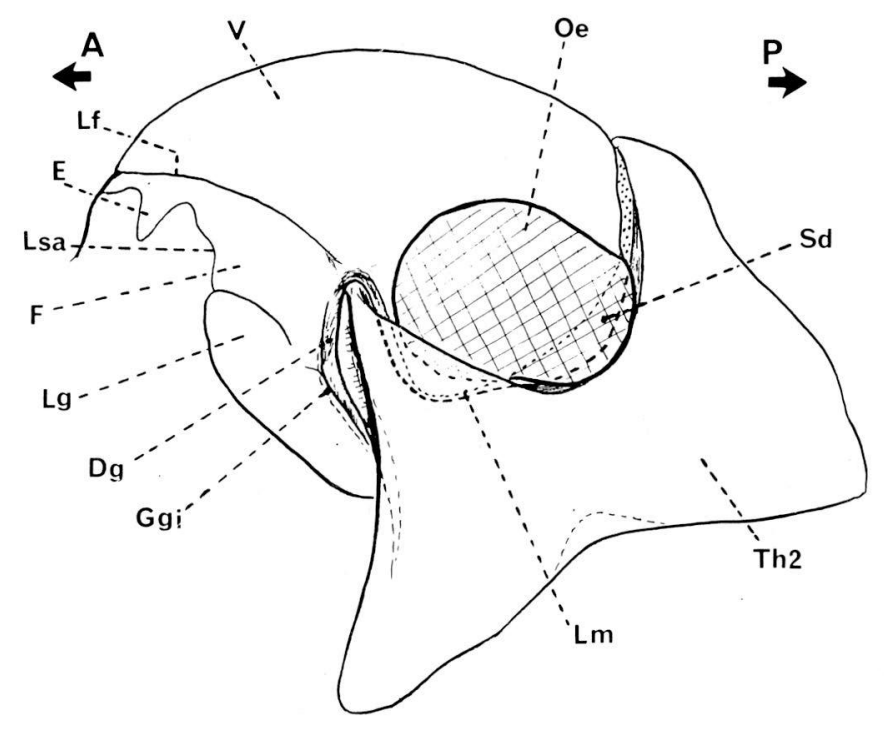

Fig. 8: Tête de Sphaeroma hookeri. Sphéromien marin oculé. Profil. Schéma expliquant la coaptation de la capsule céphalique et du péréionite 2.

2, une grande part de sa surface étant occupée par une gouttière peu profonde sur laquelle s'applique l'antenne au moment de la volvation. Il n'y a ni gouttière génale, ni semelle correspondante du deuxième segment thoracique. Quant au chevauchement alterné des bords antérieurs de ce dernier et des marges latéro-postérieures du vertex, il est remplacé par une simple butée de l'avancée thoracique sous la ligne marginale. On peut dire à peu près la même chose de Faucheria (Fig. 6).

Nous sommes donc loin de la dissociation et de l'élargissement considérable des aires génales des Sphéromiens qui, libres de tout logement des antennes, sont devenues tout entières une surface d'appui et de glissement des saillies antérieures du péréionite 2. Ce mouvement est guidé par les deux gouttières encadrant chaque dépression génale, surplombées chacune par un élément de renforcement bombé et sclérifié (Fig. I et 9). Du côté antéro-ventral, c'est 


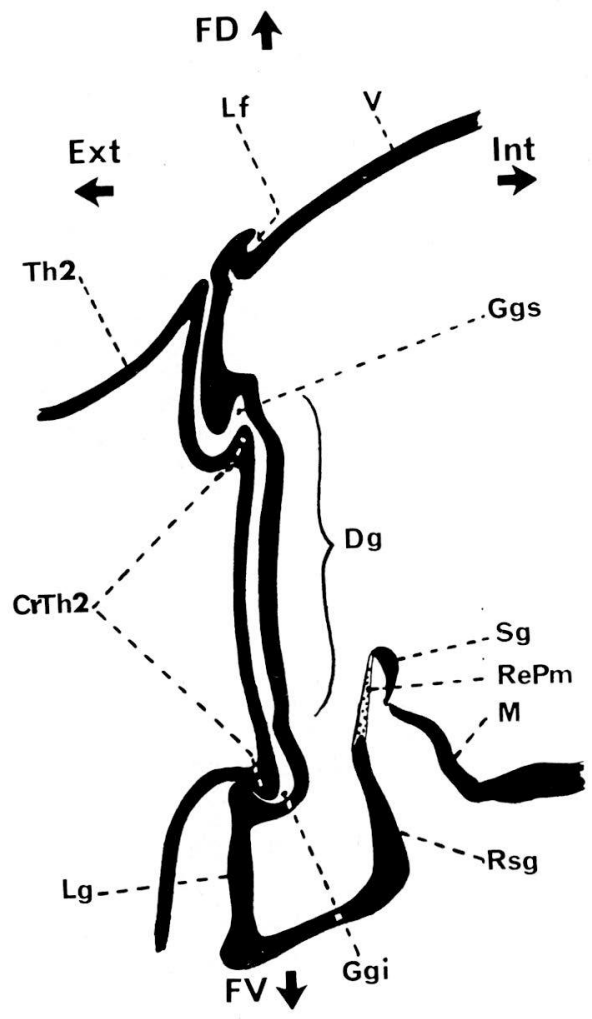

Fig. 9: Coupe transversale de la région génale de Caecosphaeroma hurgundum (portée en XY sur la figure 7). D’après une préparation microscopique.

FD: Face dorsale, FV: Face ventrale, Ext: Cöté externe, Int: Côté interne.

le lobe génal; du côté postéro-dorsal, c'est le bourrelet saillant entre les extrémités de la ligne frontale et de la ligne marginale.

\section{Conclusions}

Nous avons comparé la structure externe de la capsule céphalique de Caecosphaeroma burgundum à celle de plusieurs Isopodes volvationnels. Les différentes lignées qui ont acquis cette faculté d'enroulement l'ont fait suivant des modalités diverses, mais ont abouti à une étonnante convergence de forme sous l'influence d'exigences mécaniques précises:

- réalisation d'une boule aussi hermétique que possible par appui du pléotelson, très modifié, sur la tête, également modifiée. 
- coaptation de la tête et du péréionite 2 .

- logement des antennes et des palpes mandibulaires pendant la volvation.

Vandel $(1948,1959)$ a mis en relief, chez les Isopodes volvationnels exoantennés, l'importance capitale de ce dernier facteur dans la genèse progressive des coaptations. En effet, lorsqu'un groupe d'Isopodes permet de suivre l'évolution de la volvation au sein d'une lignée bien définie, comme celle des Spelaeoniscidae, on constate que les transformations affectent en premier lieu la région antérieure de la capsule céphalique, avec l'apparition de gouttières de plus en plus importantes. Ces modifications s'étendent ensuite progressivement à la tête tout entière, à la région antérieure de l'animal puis à l'ensemble du corps, les uropodes étant les derniers touchés par ce processus évolutif.

Bien que les conséquences en soient fonctionnellement identiques, les endoantennés examinés au cours de cette étude ont adopté, au niveau de la capsule céphalique, des solutions différentes pour aboutir à une protection des antennes aussi parfaite que possible à l'intérieur de la boule ainsi formée. Ces solutions dépendent étroitement de l'insertion des antennes sur la tête et de la direction de leur reploiement.

\section{Les quatre orbites d'insertion sont de forte taille et proches du plan sagittal}

Au niveau de la ligne médiane de la face se développe une crête verticale de séparation et de renforcement que l'écusson et le processus frontal peuvent constituer à eux seuls (cas de Faucheria).

Les antennes se replient alors de deux façons:

- Caecosphaeroma et Sphéromiens: leur reploiement se fait en direction ventrale, donc en creusant obligatoirement dans la face une gouttière médiale par rapport au lobe génal (Fig. 1). La face est enfoncée et sa surface visible de l'extérieur est rétrécie par le façonnement de ces deux profondes gouttières antennaires dont les bords se développent et engaînent les antennes repliées dans ce cadre robuste.

Tous les éléments constitutifs de la face subissent une évolution poussée dans ce sens: adjonction au processus frontal d'un énorme clypéus, grande extension du lobe génal et du repli supra-antennaire. Une conséquence importante de ce premier type de reploiement des antennes est que la géna, entièrement libre de tout logement antennaire, subit de son côté une évolution orientée exclusivement vers la coaptation avec le péréionite 2 .

- Faucheria: le reploiement des antennes est rectiligne, en direction latérale, à plat sur la face puis sur la géna, dorsalement à un lobe génal discret et sans creusement de gouttières profondes, donc sans repli supra-antennaire important et sans hypertrophie du clypéus (Fig. 6). La face, très élargie, passe sans transition aux aires génales où la place disponible est partagée entre le logement des antennes et la coaptation avec le segment thoracique 2. Dans l'ensemble, les transformations de la capsule céphalique ne sont pas aussi poussées que chez les Sphéromiens. 
Ce deuxième type de reploiement des antennes chez les Isopodes aquatiques volvationnels endoantennés ressemble à celui qu'ont adopté les Oniscoïdes supérieurs.

2. Isopodes terrestres: seules les orbites d'insertion des antennes sont de forte taille (antennules réduites). Elles sont très écartées du plan sagittal

Aucune crête médiane n'apparaît sur la face. L'écusson est discret ou absent; il n'y a ni processus frontal saillant ni clypéus important.

Les antennes se logent à plat, comme chez Faucheria, sur une face élargie, mais leur reploiement est différent. Il s'effectue d'abord en direction médiale puis, après un coude brutal, en direction latérale (voir par exemple les figures de Gruner, 1966, concernant Armadillidium). Au niveau de la géna, l'antenne oblique franchement en direction ventrale. Ici également, les gouttières antennaires sont superficielles et les aires génales sont occupées à la fois par le logement de l'antenne et l'appui du péréionite 2 .

Il est vraisemblable que ce type de reploiement des antennes ne fait qu'accentuer l'écartement de leurs insertions qui atteint son maximum chez $A r$ madillo.

Ainsi, l'évolution vers la forme volvationnelle emprunte des voies différentes pour aboutir à des capsules céphaliques d'aspect très dissemblable. Les Sphéromiens d'une part et les Oniscoïdes d'autre part en sont les représentants les plus caractéristiques. Il serait alors tentant de considérer qu'il existe parmi les Isopodes volvationnels endoantennés une évolution propre aux formes aquatiques et une autre propre aux formes terrestres. Le cas de Faucheria, Cirolanide des eaux souterraines dont la face évoque celle d'un Isopode terrestre volvationnel endoantenné, prouve qu'il n'en est rien.

Dans un autre ordre d'idées, l'étude des Spelaeoniscidae (Vandel, 1948, 1959) a permis de mettre en évidence, au sein de ce groupe, une évolution graduelle, orthogénétique, dans l'apparition des coaptations et dans leur extension progressive à l'ensemble du corps.

Dans la lignée des Cirolanides, nous l'avons déjà signalé plus haut, il est possible de suivre cette tendance évolutive au niveau du processus frontal par exemple, qui atteint son plus grand développement dans l'espèce volvationnelle Faucheria faucheri. Par contre, tous les Sphéromiens actuels sont capables d'emblée d'une volvation quasi-parfaite et seuls les uropodes présentent encore des stades évolutifs différents. Ils sont grands (Monolistra, Sphaeroma), vestigiaux (Caecosphaeroma virei) ou pratiquement nuls ( $C$. burgundum).

Si réellement telson et uropodes sont concernés en dernier lieu par les transformations liées à la volvation, on peut admettre que les Sphéromiens en sont à un stade évolutif très avancé où les coaptations au niveau de la tête ont atteint leur achèvement et où seules les dernières retouches ont lieu au niveau du pléotelson. La forme la plus évoluée est alors sans conteste Caecosphaeroma burgundum. Cet habitant des eaux souterraines réalise une sphère parfaite 

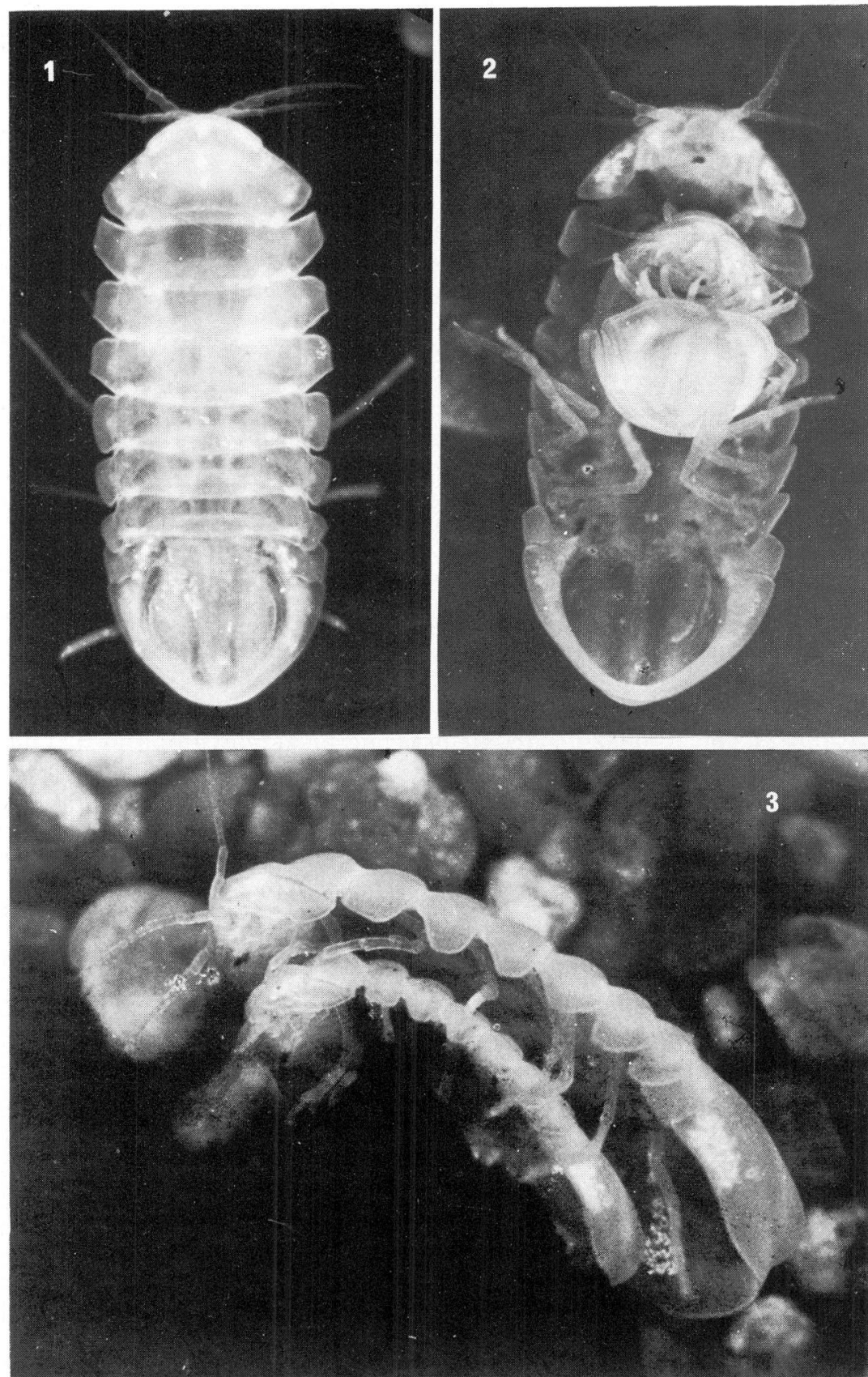
sans saillies extérieures, possède un pléotelson monolithique et sans faille dont la coaptation sur la tête et les deux premiers péréionites libres est hermétique, des gouttières antennaires profondes, un système complexe d'engrenage entre le péréionite 2 et les aires génales.

Cette adaptation de Caecosphaeroma burgundum à la volvation ne s'est pas faite entièrement dans le milieu aquatique souterrain, puisque les Sphéromiens marins, ancêtres probables des Caecosphaeroma, présentaient certainement déjà, à l'époque où ils ont pénétré dans le domaine obscuricole, des transformations bien engagées dans ce sens. C'est ce que suggère l'étude comparative des adaptations de la capsule céphalique à la volvation chez leurs représentants actuels.

L'évolution ultérieure vers une forme volvationnelle encore plus parfaite s'est alors achevée dans les eaux souterraines pendant la longue période qui sépare le début de cette colonisation de l'époque actuelle. Les Caecosphaeroma ne sont en effet probablemeni pas des animaux à vocation cavernicole récente, contrairement à ce que Vandel (1964) affirme, sur la foi des données de Paris (1925) ou des observations de Remy (1948) relatives à des individus pigmentés de Caecosphaeroma virei trouvés dans une grotte de l'Ain. Cette existence de Caecosphaeroma réellement "pigmentés" avait déjà été mise en doute dans l'étude critique de Husson (1965). Le travail de Graf et Marvillet (1966) devait démontrer que les colorations observées ça et là dans la nature ou en élevage ne doivent rien à la présence de pigments, mais qu'elles sont dues à l'agglutination de substances colorées extérieures, en surface de la carapace, pouvant aller dans certains cas jusqu'à une imprégnation de la cuticule elle-même.

On voit mal en effet comment se ferait la réacquisition de pigments par des espèces qui en sont totalement dépourvues depuis longtemps, en même temps, comme le fait remarquer Spandl (1926), qu'elles ont perdu toute trace de structures oculaires.

Vandel, dans ce même ouvrage de 1964, donne cependant pour probable que l'origine des Sphéromiens cavernicoles et de leurs Ostracodes conımensaux, comme celle des Cirolanides, doit remonter au début du tertiaire. Il est en cela tout à fait en accord avec les idées émises par Hubault (1934) et Daum (1954).

A l'appui de cette hypothèse de "l'ancienneté" de Caecosphaeroma burgundum, suggérée par la paléogéographie, viennent s'inscrire d'autres arguments:

Fig. 1: Individu mäle de Caecosphaeroma hurgundum (taille: $14 \mathrm{~mm}$ ) en face dorsale.

Fig. 2: Mâle et femelle de Caecosphaeroma hurgundum accouplés, retournés sur leur face dorsale et vus au moment où le mále ( $14 \mathrm{~mm}$ de long) s'est déroulé et montre sa lace ventrale, alors que la femelle ( $10 \mathrm{~mm}$ de long) est encore enroulée. On voit que ce fort ciimorphisme sexuel permet à la femelle d’être complètement entourée par le mále lorsque les deux individus sont enroulés.

Fig. 3: Couple de Caecosphaeroma hurgundum vu de profil. Le mále tient la femelle par ses péréiopodes 2,3 et 4 . 
Les caractères anatomiques déjà cités (dépigmentation et cécité totales, évolution poussée vers la volvation) plaident en faveur d'une longue évolution dans le milieu souterrain.

La distribution géographique actuellement connue de cette espèce montre qu'elle est radicalement coupée des faunes marines d'origine et cela correspond tout à fait à l'une des caractéristiques énoncées par Chappuis (1953) lorsqu'il définit les "espèces relictes".

Enfin, le rythme extrêmement lent des phénomènes biologiques de Caecosphaeroma burgundum (Husson et Daum, 1953 - Daum, 1954 - Marvillet, 1970) le place également parmi les cavernicoles "anciens".

\section{Quel rôle faut-il attribuer à la volvation dans le domaine aquatique souterrain?}

Pour Racovitza (1910) et bien d'autres, c'est un procédé de défense passive contre les prédateurs adopté même par des Isopodes chez lesquels la volvation n'est pas encore très évoluée. L'atrophie des uropodes, devenus inutiles dans le milieu souterrain fissuré, ne serait que la conséquence de la tendance à former une boule n'offrant aucune prise aux Amphipodes carnassiers du genre Niphargus.

Nos observations dans le milieu cavernicole nous conduisent à des conclusions plus nuancées. En eau calme, là où de nombreux Caecosphaeroma séjournent sur les feuilles ou le bois morts dont ils se nourrissent, les gros Niphargus virei n'ont aucun mal à s'en emparer, même s'ils se roulent en boule. En eau courante il est au contraire impossible à ces prédateurs aveugles d'attraper une boule qui ne reste pas sur place mais court au fil des tourbillons.

Dans l'ensemble, et dans la mesure où tout système de défense n'est toujours que relatif et ne fait que limiter les pertes au sein d'une espèce, la volvation n'est efficace chez les Isopodes des eaux souterraines que dans le cas où l'animal enroulé est emporté par le courant, tout comme un Insecte fait le mort au moment d'être saisi. Il faut rappeler également que les Cuecosphaeroma accouplés peuvent s'enrouler en deux boules parfaitement concentriques, le mâle entourant la femelle, (Figure 2 de la planche hors-texte) et que la volvation est un moyen efficace de protection des embryons chez la femelle gestante.

D'autres considèrent que la volvation est un moyen de survie en exondation temporaire (Arcangeli, 1929, cité par Vandel, 1943) ou pendant des périodes de sécheresse relative. C'est vrai pour les Isopodes adaptés à la vie terrestre qui peuvent même rester enroulés, comme au repos, aux heures sèches de la journée (Mead, 1963), mais ces variations de température et d'humidité n'interviennent pas en milieu aquatique souterrain. Si l'on trouve assez fréquemment des Caecosphaeroma émergés enroulés sur une paroi, ce n'est qu'une conséquence des variations de niveau des eaux souterraines (Daum, 1954). Encore faut-il que ces fluctuations soient temporaires, sinon l'animal a peu de chances de survie.

A notre avis la volvation a une conséquence indirecte, des plus importantes 
dans le peuplement du milieu souterrain. C'est un mode de dissémination passive rapide, très efficace, auquel les Caecosphaeroma sont parfaitement adaptés. Il suffit pour s'en convaincre de voir avec quelle agilité un individu enroulé et entraîné par le courant peut se dérouler et s’agripper en une fraction de seconde à tout nouveau support qui vient à sa portée. Dans les réseaux de fissures souterraines où ils vivent, c'est évidemment, pour ces Isopodes marcheurs et lents à se déplacer, une façon de parcourir des distances relativement importantes en un temps bref.

Il n'est cependant pas exclu que l'acquisition de la volvation corresponde à des évolutions orthogénétiques parallèles sans utilité immédiate (Vandel, 1943). C'est une hypothèse à ne pas perdre de vue, mais nous devons constater que, dans les eaux du domaine qu'ils peuplent, les Isopodes aquatiques volvationnels utilisent au mieux les possibilités que leur offre ce dispositif anatomique.

\section{RÉSUMÉ}

L'étude de la capsule céphalique de l'Isopode des eaux souterraines Caecosphaeroma burgundum révèle des adaptations extrêmement poussées à la volvation qui intéressent également les autres régions du corps, en particulier le pléotelson.

Chez ce Sphéromien aveugle, à partir d'une structure encore primitive d'Isopode aquatique, la tête a été entièrement modelée en fonction de plusieurs impératifs mécaniques: l'appui du bord postérieur du pléotelson, l'engrenage des extrémités antérieures du deuxième péréionite et, surtout, le logement des palpes mandibulaires et des antennes dans deux gouttières profondes de la face.

L'importance de ce "facteur antennaire" est mise en évidence par l'étude comparative de la tête d'autres Isopodes volvationnels: des Oniscoïdes, des Sphéromiens épigés et d'autres Isopodes des eaux souterraines (deux Sphéromiens et un Cirolanide). Cette comparaison montre en même temps que Caecosphaeroma burgundum est certainement le plus hautement spécialisé de tous et qu'il approche la perfection dans la volvation car lui seul s'enroule en une sphère hermétique sans saillies extérieures.

Le rôle de la volvation apparaît double. C'est un moyen de défense contre les prédateurs, utilisé aussi bien par les animaux isolés que par les couples, $\hat{\delta}$ et o étant alors associés en deux boules concentriques. C'est aussi un mode de dissémination passive très important dans le peuplement à distance des eaux souterraines.

Légendes des figures

Al: Antenne 1

A2 : Antenne 2 
adM : Insertion de l'adducteur mandibulaire

Ap : Apodème latéro-ventral de la capsule céphalique

ApA1: Apodème articulaire de l'antenne 1

ApA2: Apodème articulaire de l'antenne 2

$\mathrm{Cl}:$ Clypéus

CIPl : Processus latéral du clypéus

Crmt : Crête intermandibulaire transverse

$\mathrm{Dg}$ : Dépression génale

DTh2: Dépression du bord antérieur du péréionite 2

E : Ecusson

F : Front

Fo : Foramen occipital

G : : Gena

Ggi : Gouttière inférieure de la dépression génale

Ggs : Gouttière supérieure de la dépression génale

GPM : Gouttière du Palpe mandibulaire

imA2 : Insertion des muscles de l'antenne

iTld : Insertion du bras dorsal du tentorium antérieur

LA : Labre

Laf : Lamina frontale

Lf : Ligne frontale

$\mathrm{Lg}$ : Lobe génal

Lm : Ligne marginale

Lsa : Ligne supra-antennaire

m : membrane

M : Mandibule

Maa : Articulation antérieure de la mandibule

Map : Articulation postérieure de la mandibule

Oe : Oeil

OA1 : Orbite d'insertion de l'antenne 1

OA2 : Orbite d'insertion de l'antenne 2

Pf : Processus frontal

PG : Postgena

PL : Pléotelson

PM :- Palpe mandibulaire

RePm: Renforcement externe de la poche articulaire de la mandibule

Rsa : Repli supra-antennaire

Rsap : Limite postérieure du repli supra-antennaire

Rsg : Repli sub-génal

$\mathrm{Sd}$ : Surplomb latéro-dorsal du vertex

$\mathrm{Sg}$ : Sclérite glénoïde

T2 : Tentorium postérieur

Th2 : Péréionite 2

Th3 : Péréionite 3

Ur : Uropode

V : Vertex 


\section{BIBLIOGRAPHIE}

BERTRAND, J. Y. (1974) - Recherches sur l'écologie de Fancheria fancheri (Crustacés, Cirolanides). Thèse Univ. Paris VI, $123 \mathrm{p}$.

CHAPPUIS, P. A. (1953) - Sur certaines reliques marines dans les eaux souterraines. C.R. Ier Congr. Intern. Spel. Paris, 47-53.

CHAUDONNERET, J. (1967) - Méthodes danatomie morphologique. Ann. Soc. Entom. Fr, 3. 3-11.

DAUM. J. (1954) - Zur Biologie einer Isopodenart unterirdischer Gewässer: Caecosphacroma burgundum Dollfus. Ann. Univers. Sarav., Naturwiss., 3, 104-160.

GRAF, F. et MARVILLET, C. (1966) - Sur la faculté d’absorption de substances colorées par la cuticule de Caecosphaeroma burgundum Dollfus, Crustacé Isopode des eaux souterraines. Int. J. Spel., 2, 335-339.

GRUNER. H. E. (1953) - Der Rollmechanismus bei kugelnden Landisopoden und lsopoden. Mitt. Zool. Mus. Berlin, 29, 148-179.

GRUNER, H. E. (1965-1966) - Krebstiere oder Crustacea, V, Isopoda. Tierwelt Deutschlands, 51 et 53 , Jena, $380 \mathrm{p}$.

HENNEQUIN, G. (1934) - Mécanisme denroulement de Sphaeroma serratum. Trav. Stat. Biol. Roscoff, 12, 23-30.

HOLMGREN, N. (1920) - Vergleichendes über den Kopfbau der Crustaceen und Hexapoden. Ark. für Zool., 13.

HOLTHUIS, L. B. (1956) - Isopoda en Tanaidacea (KV). Fauna van Nederland. I6. Leiden, $280 \mathrm{p}$.

HUBAULT. E. (1934) - Etude faunistique d’eaux souterraines à la lisière septentrionale du bassin d'Aquitaine. Bull. Biol. Fr. et Belg., 68, 59-73.

HUSSON. R. et DAUM. J. (1953) - Sur la biologie de Caecosphaeroma hurgumdum. C.R. Ac. Sc. Paris, 236, 2345-2347.

HUSSON, R. (1969) - Existence d'individus pigmentés dans l'espèce troglobie Caecosphacroma burgundum Dollfus (Crustacea - Isopoda). Actes IV Congr. Intern. Spel.. Yougoslavie, $1965,125-128$.

JACKSON, H. G. (1926) - The morphology of the Isopod head. Part I. The head or ligia oceanica. Proc. Zool. Soc. London, 885-910.

JACKSON, H. G. (1928) - The morphology of the lsopod head. Part Il. The terrestrial Isopods. Proc. Zool. Soc. London, 561-595.

LOMBARDO, C. A. (1975) - Morfologia del dermascheletro del capo di Anilocra phlyodes I. (Crustacea, Isopoda). Cah. Biol. Mar., 16, 301-316.

MANTON, S. (1964) - Mandibular mechanisms and the evolution of Arthropods. Phil. Trans. Roy. Soc. London, B, 247, 1-183.

MARVILLET, C. (1970) - Quelques données nouvelles sur la biologie de Caecosphaeroma hurgundum Dollfus. Crustacé Isopode des eaux souterraines. Spelunca, 7, 137-140.

MARVILLET, C. (1972) - Le squelette sternal gnatho-céphalique de quelques Crustacés Isopodes. Bull. Soc. Zool. Fr., 97, 183-195.

MARVILLET, C. et CHAUDONNERET, J. (1975) - L'articulation postérieure de ia mandibule chez Caecosphaeroma hurgundum Dollfus, Isopode volvationnel des eaux souterraines. C. R. Ac. Sc. Paris, 281, 407-410.

MEAD, F. (1963) - Recherches sur l'écologie et le comportement d'un Isopode terrestre: Helleria brevicornis Ebner. Thèse Fac. Sc. Marseille, 78 p.

RACOVITZA, E. G. (1910) - Sphéromiens ( ${ }^{\circ}$ série) et révision des Monolistrini (Isopodes Sphéromiens). Biospeologica, 13. Arch. Zool. exp. gén., (5), 4, 625-758.

RACOVITZA, E. G. (1912) - Cirolanides ( $1^{\circ}$ série). Biospeologica, 27. Arch. Zool. exp. gén., (5), 10, 203-329.

SCHMALFUSS, H. (1974) - Skelett und Extremitäten-Muskulatur des Isopoden-Cephalothorax. Z. Morph. Tiere, 78, 1-91.

SNODGRASS, R. E. (1952) - A textbook of Arthropod anatomy. Ithaca, N. York. 363 p. SPANDL, H. (1926) - Die Tierwelt der unterirdischen Gewässer. Spel. Inst.. Wien. 235 p. 
TAIT, J. (1917) - Experiments and observations on Crustacea. Pt IV. Some structural features pertaining to Glyptonotus. Proc. Roy. Soc. Edinburgh, 37, $\mathrm{n}^{\circ} 15,246-303$.

VANDEL, A. (1943) - Essai sur l'origine, l'évolution et la classification des Oniscoidea (Isopodes terrestres). Bull. Biol. Fr. et Belg., suppl. 30, 1-136.

VANDEL. A. (1948) - - Les Isopodes volvationnels exoantennés et la genèse de leurs coaptations. Bull. Biol. Fr. et Belg., 82, 388-428.

VANDEL, A. (1959) - Nouvelles recherches sur les Isopodes volvationnels exoantennés et la genèse de leurs coaptations. Bull. Biol. Fr. et Belg., 93, 121-139.

VANDEL, A. (1960-1962) - Isopodes terrestres ( $1^{\circ}$ et $2^{\circ}$ parties). Faune de France, 64 et 66 , Lechevalier, Paris, 931 p.

VANDEL, A. (1964) -- Biospéologie. Gauthier-Villars, Paris, 619 p. 